\title{
A NON-ABELIAN TENSOR PRODUCT OF LIE ALGEBRAS
}

\author{
by GRAHAM J. ELLIS
}

(Received 19 June, 1989; revised 8 December, 1989)

0. Introduction. A generalized tensor product of groups was introduced by $\mathbf{R}$. Brown and J.-L. Loday [6], and has led to a substantial algebraic theory contained essentially in the following papers: $[6,7,1,5,11,12,13,14,18,19,20,23,24]([9,27,28]$ also contain results related to the theory, but are independent of Brown and Loday's work). It is clear that one should be able to develop an analogous theory of tensor products for other algebraic structures such as Lie algebras or commutative algebras. However to do so, many non-obvious algebraic identities need to be verified, and various topological proofs (which exist only in the group case) have to be replaced by purely algebraic ones. The work involved is sufficiently non-trivial to make it interesting.

In this article we have chosen to work with Lie algebras. We introduce a non-abelian tensor product of Lie algebras and investigate its properties. In particular we study its relation to the low dimensional homology of Lie algebras. (A few results on this Lie tensor product have been obtained previously $[10,15,21]$.)

Many of the results in this article are analogues of known group theoretic results. However the proofs are often quite different. Several of our proofs convert to new proofs of corresponding group theoretic results. In particular our proof of Theorem 24 yields, using [17], a new proof of the main result of [30] which avoids intricate elementwise calculations. Our proof of Proposition 19 yields a new proof of the main result of [18] which again avoids many intricate elementwise computations. Our proof of Theorem 18 yields an algebraic proof of a corresponding group theoretic result (see [13]), thus solving a problem posed in [5].

1. The tensor product. Let $\Lambda$ be a commutative ring with identity. We shall use the term Lie algebra to mean a Lie algebra over $\Lambda$; we shall use [,] to denote the Lie bracket.

Let $M$ and $P$ be two Lie algebras. By an action of $P$ on $M$ we mean a $\Lambda$-bilinear map $P \times M \rightarrow M,(p, m) \mapsto^{p} m$ satisfying

$$
\begin{aligned}
{\left[p, p^{\prime}\right]_{m} } & ={ }^{p}\left({ }^{P^{\prime}} m\right)-{ }^{p^{\prime}}\left({ }^{P} m\right), \\
{ }^{p}\left[m, m^{\prime}\right] & =\left[{ }^{p} m, m^{\prime}\right]+\left[m,{ }^{P} m^{\prime}\right],
\end{aligned}
$$

for all $m, m^{\prime} \in M, p, p^{\prime} \in P$. For example, if $P$ is a subalgebra of some Lie algebra $Q$ (maybe $P=Q$ ), and if $M$ is an ideal in $Q$, then Lie multiplication in $Q$ yields an action of $P$ on $M$.

Suppose that $M$ and $N$ are Lie algebras with an action of $M$ on $N$ and action of $N$ on $M$. For any Lie algebra $Q$ we call a bilinear function $h: M \times N \rightarrow Q$ a Lie pairing if

$$
\begin{aligned}
h\left(\left[m, m^{\prime}\right], n\right) & =h\left(m,{ }^{m^{\prime}} n\right)-h\left(m^{\prime},{ }^{m} n\right), \\
h\left(m,\left[n, n^{\prime}\right]\right) & =h\left({ }^{n^{\prime}} m, n\right)-h\left({ }^{n} m, n^{\prime}\right), \\
h\left({ }^{n} m,{ }^{m^{\prime}} n^{\prime}\right) & =-\left[h(m, n), h\left(m^{\prime}, n^{\prime}\right)\right],
\end{aligned}
$$

for all $m, m^{\prime} \in M, n, n^{\prime} \in N$. For example if $M$ and $N$ are both ideals of some Lie algebra then the function $M \times N \rightarrow M \cap N,(m, n) \mapsto[m, n]$ is a Lie pairing.

A Lie pairing $h: M \times N \rightarrow Q$ is said to be universal if for any other Lie pairing

Glasgow Math. J. 33 (1991) 101-120. 
$h^{\prime}: M \times N \rightarrow Q^{\prime}$ there is a unique Lie homomorphism $\theta: Q \rightarrow Q^{\prime}$ such that the diagram

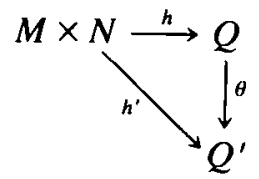

commutes. Clearly if $h$ is universal then $Q$ is determined up to isomorphism by $M, N$ and the actions. In fact $Q$ can be described as a "tensor product".

Definition. The tensor product $M \otimes N$ is the Lie algebra generated by the symbols $m \otimes n$ (for $m \in M, n \in N$ ) subject only to the relations:

(i) $\lambda(m \otimes n)=\lambda m \otimes n=m \otimes \lambda n$,

(ii) $\left(m+m^{\prime}\right) \otimes n=m \otimes n+m^{\prime} \otimes n$, $m \otimes\left(n+n^{\prime}\right)=m \otimes n+m \otimes n^{\prime}$,

(iii) $\left[m, m^{\prime}\right] \otimes n=m \otimes\left({ }^{\prime} n\right)-m^{\prime} \otimes\left({ }^{m} n\right)$, $m \otimes\left[n, n^{\prime}\right]=\left({ }^{n^{\prime}} m\right) \otimes n-\left({ }^{n} m\right) \otimes n^{\prime}$,

(iv) $\left[(m \otimes n),\left(m^{\prime} \otimes n^{\prime}\right)\right]=-\left({ }^{n} m\right) \otimes\left(m^{\prime} n^{\prime}\right)$

for $\lambda \in \Lambda, m, m^{\prime} \in M, n, n^{\prime} \in N$.

Proposition 1. The mapping

$$
h: M \times N \rightarrow M \otimes N,(m, n) \mapsto m \otimes n
$$

is a universal Lie pairing.

To be able to say much about the tensor product $M \otimes N$ we need to assume that the action of $M$ on $N$ is compatible, in the following sense, with the action of $N$ on $M$.

Definition. The actions are compatible if

$$
{ }^{\left({ }^{n} m\right)} n^{\prime}=\left[n^{\prime},{ }^{m} n\right] \text { and }{ }^{\left({ }^{m} n\right)} m^{\prime}=\left[m^{\prime},{ }^{n} m\right]
$$

for all $m, m^{\prime} \in M, n, n^{\prime} \in N$.

From this point on we assume that the actions of $M$ and $N$ are compatible. This is the case, for example, if $M$ and $N$ are both ideals of some Lie algebra with the actions given by Lie multiplication.

Proposition 2. There are two Lie homorphisms

$$
\mu: M \otimes N \rightarrow M \quad \text { and } \quad v: M \otimes N \rightarrow N
$$

defined on generators by $\mu(m \otimes n)=-{ }^{n} m$ and $v(m \otimes n)={ }^{m} n$.

We shall denote by $[M, N]^{N}$ or $[N, M]^{N}$ the submodule of $N$ generated by the elements of the form ${ }^{m} n$ with $m \in M, n \in N$. It follows from the compatibility condition that $[M, N]^{N}$ is an ideal of $N$.

Recall from [26] that, in the context of Lie algebras, a crossed module is a Lie homomorphism $\partial: M \rightarrow P$ together with an action of $P$ on $M$ such that

(i) $\partial\left({ }^{p} m\right)=[p, \partial m]$,

(ii) ${ }^{(\partial m)} m^{\prime}=\left[m, m^{\prime}\right]$

for all $m, m^{\prime} \in M, p \in P$. 
As another instance of compatible actions, note that if $\partial: M \rightarrow P$ and $\partial^{\prime}: M^{\prime} \rightarrow P$ are two crossed modules then $M$ and $M^{\prime}$ act on each other via the action of $P$. These actions are compatible.

There are many examples of crossed modules. We list a few here. Throughout, $P$ denotes an arbitrary Lie algebra.

(1) If $M$ is an ideal in $P$ then the inclusion $M \hookrightarrow P$ is a crossed module.

(2) If $M$ is a $P$-module (i.e. an abelian Lie algebra with a $P$-action) then the trivial homomorphism $M \stackrel{0}{\rightarrow} P$ is a crossed module.

(3) Any surjective Lie homomorphism $M \rightarrow P$ whose kernel lies in the centre of $M$ is a crossed module. In this case $p \in P$ acts on $m \in M$ by ${ }^{p} m=[\tilde{p}, m]$ where $\tilde{p} \in M$ is any element in the pre-image of $p$.

(4) If $P=\operatorname{aut}(M)$ is the Lie algebra of derivations on some Lie algebra $M$, then the homomorphism $M \rightarrow P$ which sends an element $m \in M$ to the corresponding inner derivation $M \rightarrow M, m^{\prime} \mapsto\left[m, m^{\prime}\right]$ is a crossed module.

The next proposition is taken from [10]. A published proof can be found in [21].

Proposition 3. There are actions of both $M$ and $N$ on $M \otimes N$ given by

$$
\begin{aligned}
m^{\prime}(m \otimes n) & =\left[m^{\prime}, m\right] \otimes n+m \otimes\left(m^{\prime} n\right), \\
{ }^{\prime}(m \otimes n) & =\left({ }^{n^{\prime}} m\right) \otimes n+m \otimes\left[n^{\prime}, n\right]
\end{aligned}
$$

for $m, m^{\prime} \in M, n, n^{\prime} \in N$. With these actions the homomorphisms $\mu: M \otimes N \rightarrow M$ and $v: M \otimes N \rightarrow N$ are crossed modules.

It is worth noting that for any crossed module $\partial: M \rightarrow P$ the image of $\partial$ is an ideal in $P$, and hence we can form the quotient Lie algebra $P / \partial(M)$. Also, the kernel of $\partial$ is a $P$-invariant ideal in the centre of $M$, and thus in particular is abelian. Moreover the action of $P$ on $\operatorname{ker}(\partial)$ induces an action of $P / \partial(M)$ on $\operatorname{ker}(\partial)$, making $\operatorname{ker}(\partial)$ a $P / \partial(M)$-module.

So for the crossed modules $\mu$ and $\nu$ of the last proposition, we get that $\operatorname{ker}(\mu)$ is an $M /[M, N]^{M}$-module, and that $\operatorname{ker}(v)$ is an $N /[M, N]^{N}$-module.

There is a useful relation between our Lie tensor product $M \otimes N$ and the standard tensor product of $M$ and $N$ considered as $\Lambda$-modules. We denote this latter tensor product by $M \bigotimes_{\bmod } N$. Recall that $M \bigotimes_{\text {mod }} N$ is the $\Lambda$-module generated by symbols $m \otimes n$ (for $m \in M$, $n \in N)$ subject to the relations:

$$
\begin{aligned}
\lambda(m \otimes n) & =\lambda m \otimes n=m \otimes \lambda n, \\
\left(m+m^{\prime}\right) \otimes n & =m \otimes n+m^{\prime} \otimes n, \\
m \otimes\left(n+n^{\prime}\right) & =m \otimes n+m \otimes n^{\prime},
\end{aligned}
$$

for $\lambda \in \Lambda, m, m^{\prime} \in M, n, n^{\prime} \in N$.

Proposition 4. There is a $\Lambda$-module surjection

$$
M \bigotimes_{\bmod } N \rightarrow M \otimes N, m \otimes n \mapsto m \otimes n
$$

Proof. The map is clearly a $\Lambda$-module homomorphism. It is surjective by virtue of identity (iv) of the Lie tensor product.

Sometimes the surjection of Proposition 4 is an isomorphism. 
Proposition 5. If $M$ and $N$ act trivially on each other (i.e. $[M, N]^{M}=\{0\}$, $\left.[M, N]^{N}=\{0\}\right)$ then there is an isomorphism

$$
M \otimes N \cong\left(M^{a b}\right) \bigotimes_{\bmod }\left(N^{a b}\right),
$$

where $M^{a b}=M /[M, M]$ and $N^{a b}=N /[N, N]$.

Proof. Here identity (iii) of the Lie tensor product states that $\left[m, m^{\prime}\right] \otimes n=0$ and $m \otimes\left[n, n^{\prime}\right]=0$ for all $m, m^{\prime} \in M, n, n^{\prime} \in N$.

The Lie tensor product $\otimes$ is not associative. It is however symmetric and it is distributive over certain direct sums, as we now show.

Proposition 6. There is an isomorphism

$$
M \otimes N \cong N \otimes M, \quad m \otimes n \mapsto-n \otimes m .
$$

Proof. It is readily checked that $h: M \times N \rightarrow N \otimes M,(m, n) \mapsto n \otimes m$ is a Lie pairing. The universal property of $M \otimes N$ thus yields a homomorphism $M \otimes N \rightarrow N \otimes M$. There is similarly an (inverse) homomorphism $N \otimes M \rightarrow M \otimes N$.

Lemma 7. Suppose that $L, M, N$ are Lie algebras such that

(i) $L$ and $N$ act compatibly on one another, and $M$ and $N$ act compatibly on one another;

(ii) ${ }^{l}\left({ }^{m} n\right)={ }^{m}\left({ }^{l} n\right)$ for all $l \in L, m \in M, n \in N$;

(iii) the canonical homomorphisms $[L, N]^{N} \otimes M \rightarrow N \otimes M$ and $[M, N]^{N} \otimes L \rightarrow$ $N \otimes L$ are trivial.

Then the direct sum of Lie algebras $L \oplus M$ acts on $N$ by ${ }^{(l, m)} n={ }^{\prime} n+{ }^{m} n$, and $N$ acts on $L \oplus M$ by ${ }^{n}(l, m)=\left({ }^{n} l,{ }^{n} m\right)$. With these Lie actions there is an isomorphism

$$
(L \oplus M) \otimes N \cong(L \otimes N) \oplus(M \otimes N) .
$$

Proof. The actions are clearly Lie actions. It is routine to show that

$$
\alpha:(L \oplus M) \otimes N \rightarrow(L \otimes N) \oplus(M \otimes N), \quad(l, m) \otimes n \mapsto(l \otimes n, m \otimes n)
$$

yields a Lie homomorphism. The inclusions of $L$ and $M$ into $L \oplus M$ yield homomorphisms $L \otimes N \rightarrow(L \oplus M) \otimes N$ and $M \otimes N \rightarrow(L \oplus M) \otimes N$ which combine to give an inverse to $\alpha$.

Any Lie algebra $P$ acts on itself by Lie multiplication and so we can consider the tensor square $P \otimes P$. Lemma 7 provides a description of $P \otimes P$ for $P=P_{1} \oplus P_{2}$ a direct sum of Lie algebras.

Proposition 8. There is an isomorphism

$$
\left(P_{1} \oplus P_{2}\right) \otimes\left(P_{1} \oplus P_{2}\right) \cong\left(P_{1} \otimes P_{1}\right) \oplus\left(P_{1} \otimes P_{2}\right) \oplus\left(P_{2} \otimes P_{1}\right) \oplus\left(P_{2} \otimes P_{2}\right) .
$$

Proof. Note that $P_{1}$ and $P_{2}$ act on each other trivially. So we can apply Lemma 7 twice.

The tensor product $\otimes$ is functorial in some sense. To make this precise let $\mathscr{L}_{i}{ }^{2}$ be the category whose objects are ordered pairs of Lie algebras $(M, N)$ which act compatibly on each other; the morphisms of $\mathscr{L}_{i}{ }^{2}$ are pairs of Lie homomorphisms $\phi: M \rightarrow M^{\prime}$, 
$\psi: N \rightarrow N^{\prime}$ such that $\phi\left({ }^{n} m\right)={ }^{(\psi n)}(\phi M)$ and $\psi\left({ }^{m} n\right)={ }^{(\phi m)}(\psi n)$ for all $m \in M, n \in N$. Then $\otimes$ is a functor from $\mathscr{L}_{i e^{2}}$ into the category of Lie algebras. if

In $\mathscr{L}_{i}{ }^{2}$ we have the obvious notions of kernel, image and exact sequence. Note that

$$
(0,0) \rightarrow(K, L) \rightarrow(M, N) \rightarrow(P, Q) \rightarrow(0,0)
$$

is an exact sequence, then the action of $M$ on $N$ restricts to an action of $M$ on $L$, and also to an action of $K$ on $N$. Similarly there is an action of $N$ on $K$ and of $L$ on $M$. By Proposition 3 there is a homomorphism $M \otimes L \rightarrow L$ and an action of $N$ on $K \otimes N$; there is thus an action of $M \otimes L$ on $K \otimes N$, from which we can form the semidirect product $(K \otimes N) \rtimes(M \otimes L)$

Proposition 9. A short exact sequence

$$
(0,0) \rightarrow(K, L) \rightarrow(M, N) \rightarrow(P, Q) \rightarrow(0,0)
$$

in $\mathscr{L}_{i e^{2}}$ gives rise to an exact sequence of Lie algebras

$$
(K \otimes N) \rtimes(M \otimes L) \stackrel{\alpha}{\rightarrow} M \otimes N \stackrel{\beta}{\rightarrow} P \otimes Q \rightarrow 0 .
$$

Proof. Clearly $\beta$ is surjective. Letting $\alpha_{1}: K \otimes N \rightarrow M \otimes N$ and $\alpha_{2}: M \otimes L \rightarrow M \otimes N$ be the functorial homomorphisms we set $\alpha(v, w)=\alpha_{1}(v)+\alpha_{2}(w)$. Certainly $\alpha$ is a module homomorphism; the Lie bracket is preserved by $\alpha$ since, by Proposition 3 , ${ }^{(m n}\left(m^{\prime} \otimes n^{\prime}\right)=\left[m \otimes n, m^{\prime} \otimes n^{\prime}\right]$ for all $m \otimes n, m^{\prime} \otimes n^{\prime} \in M \otimes N$. The image of $\alpha$ is generated by all $k \otimes n, m \otimes l$ with $k \in K, l \in L, m \in M, n \in N$. Clearly $\beta \alpha$ is the trivial homomorphism. Now $\operatorname{Im}(\alpha)$ is an ideal in $M \otimes N$ since we have $\left[m \otimes n, k \otimes n^{\prime}\right]=$ $\left({ }^{\left({ }^{n} n\right)} k\right) \otimes n^{\prime}+k \otimes\left[{ }^{m} n, n^{\prime}\right]$ and $\left[m \otimes n, m^{\prime} \otimes l\right]=\left({ }^{\left(m_{n}\right)} m^{\prime}\right) \otimes l+m^{\prime} \otimes\left[{ }^{m} n, l\right]$. To finish the proof, it suffices to check that $h: P \times Q \rightarrow(M \otimes N) / \operatorname{Im}(\alpha),(p, q) \mapsto \tilde{p} \otimes \tilde{q}+\operatorname{Im}(\alpha)$, with $\tilde{p} \in M$ and $\bar{q} \in N$ being any elements in the pre-images of $p$ and $q$ respectively, is a well-defined Lie pairing; the universal property of the tensor product then implies that $h$ induces a homomorphism $P \otimes Q \rightarrow(M \otimes N) / \operatorname{Im}(\alpha)$ for which there is an inverse homomorphism induced by $\beta$.

One easy consequence of Proposition 9 is that if $K$ is an ideal in the Lie algebra $M$ then there is an exact sequence

$$
(M \otimes K) \rtimes(K \otimes M) \rightarrow M \otimes M \rightarrow(M / K) \otimes(M / K) \rightarrow 0 .
$$

If moreover $K$ lies in the centre of $M$ then the semi-direct product $\rtimes$ is just the direct $\operatorname{sum} \oplus$.

We end this section by showing how the tensor product is related to universal central extensions. (Recall that a central extension

$$
0 \rightarrow L \rightarrow U \stackrel{\varphi}{\rightarrow} P \rightarrow 0
$$

of $P$ is universal if for any other central extension

$$
0 \rightarrow K \rightarrow M \stackrel{\Phi}{\rightarrow} P \rightarrow 0
$$

there is a unique homomorphism $\theta: U \rightarrow M$ such that $\phi \theta=\varphi$. It is well-known that $P$ has a universal central extension if and only if $P$ is perfect, i.e. $P=[P, P]$. It is also 
well-known that if

$$
0 \rightarrow L \rightarrow U \stackrel{\varphi}{\rightarrow} P \rightarrow 0
$$

is a universal central extension, then $L$ is isomorphic to $H_{2}(P)$, the second homology of $P$ with coefficients in $\Lambda$.)

Lemma 10. If $0 \rightarrow K \stackrel{\leftrightarrow}{\rightarrow} M \stackrel{\phi}{\rightarrow} P \rightarrow 0$ is a central extension, then there is a Lie homomorphism $\xi: P \otimes P \rightarrow M$ such that $\phi \xi\left(p \otimes p^{\prime}\right)=\left[p, p^{\prime}\right]$. Moreover, if $P$ is perfect then $\xi$ is unique.

Proof. Since $K$ is in the centre of $M$ we get a Lie pairing $h: P \times P \rightarrow M$, $\left(p, p^{\prime}\right) \mapsto\left[\tilde{p}, \tilde{p}^{\prime}\right]$ where $\tilde{p}$ and $\tilde{p}^{\prime}$ are any elements in $\phi^{-1}(p)$ and $\phi^{-1}\left(p^{\prime}\right)$ respectively. This induces $\xi$. If $\xi, \xi^{\prime}: P \otimes P \rightarrow M$ are two homomorphisms such that $\phi \xi=\phi \xi^{\prime}$ then $\xi-\xi^{\prime}=\imath \eta$, where $\eta: P \otimes P \rightarrow K$ is a homomorphism which factors through the projection $P \otimes P \rightarrow(P \otimes P)^{\text {ab }}$. By relation (iv) of the tensor product, if $P$ is perfect then so is $P \otimes P$. The uniquenes of $\xi$ for perfect $P$ follows.

This lemma give us

THEOREM 11. If $P$ is a perfect Lie algebra then $P \otimes P \rightarrow P$ is the universal central extension of $P$, and hence $H_{2}(P) \cong \operatorname{ker}(P \otimes P \rightarrow P)$.

2. An exterior product and universal quadratic functor. Suppose that $\partial: M \rightarrow P$ and $\partial^{\prime}: N \rightarrow P$ are two crossed modules. In this context we say that a Lie pairing $h: M \times N \rightarrow Q$ is an exterior Lie pairing if $h(m, n)=0$ whenever $\partial(m)=\partial^{\prime}(n)$. For instance, if $\partial$ and $\partial^{\prime}$ are just inclusions of ideals, then the Lie pairing $h: M \times N \rightarrow M \cap N$, $(m, n) \mapsto[m, n]$ is exterior.

Replacing "Lie pairing" by "exterior Lie pairing" in our definition of a universal Lie pairing, we obtain the notion of a universal exterior Lie pairing.

Let $M \square N$ be the submodule of $M \otimes N$ generated by the elements $m \otimes n$ with $\partial(m)=\partial^{\prime}(n)$. Note that $M \square N$ lies in the centre of $M \otimes N$ since for any $m \otimes n \in M \square N$ and $m^{\prime} \otimes n^{\prime} \in M \otimes N$ we have

$$
\begin{aligned}
{\left[m^{\prime} \otimes n^{\prime}, m \otimes n\right] } & =-\left({ }^{n^{\prime}} m^{\prime}\right) \otimes\left({ }^{m} n\right) \\
& =-\left({ }^{n^{\prime}} m^{\prime}\right) \otimes\left({ }^{(2 m)} n\right) \\
& =-\left({ }^{n^{\prime}} m^{\prime}\right) \otimes\left({ }^{\left({ }^{\prime} n\right)} n\right) \\
& =-\left({ }^{n^{\prime}} m^{\prime}\right) \otimes[n, n] \\
& =-\left({ }^{n^{\prime}} m^{\prime}\right) \otimes 0 \\
& =0 .
\end{aligned}
$$

In particular, $M \square N$ is an ideal.

Definition. The exterior product $M \wedge N$ of $M$ and $N$ is the quotient

$$
M \wedge N=(M \otimes N) /(M \square N) ;
$$

for $m \otimes n \in M \otimes N$ we denote the coset $m \otimes n+M \square N$ by $m \wedge n$.

Proposition 12. The mapping

$$
h: M \times N \rightarrow M \wedge N, \quad(m, n) \mapsto m \wedge n
$$

is a universal exterior pairing. 
It is readily seen that Propositions 2,3 , and 6 hold with $\otimes$ replaced by $\wedge$. For any Lie algebra $P$ we can construct $P \wedge P$ from the identity map $P \rightarrow P$ (which is a crossed module). Theorem 11 then holds with $\otimes$ replaced by $\wedge$, since for a perfect Lie algebra $P$ we have $P \otimes P \cong P \wedge P$. (This isomorphism follows from identity (iv) of the Lie tensor product.)

Note that for any $p, q \in P$ we have $(p+q) \wedge(p+q)=0$ in $P \wedge P$ and, as a consequence, $p \wedge q=-q \wedge p$. This last identity implies that the exterior analogue of Proposition 8 is an isomorphism

$$
\left(P_{1} \oplus P_{2}\right) \wedge\left(P_{1} \oplus P_{2}\right) \cong\left(P_{1} \wedge P_{1}\right) \oplus\left(P_{1} \otimes P_{2}\right) \oplus\left(P_{2} \wedge P_{2}\right)
$$

when $P=P_{1} \oplus P_{2}$. Here, as in Proposition $8, P_{1} \otimes P_{2} \cong P_{1}^{a b} \bigotimes_{\text {mod }} P_{2}^{\text {ab }}$.

As a special case of the exterior analogue of Proposition 9 we have that a short exact sequence

$$
0 \rightarrow K \rightarrow M \rightarrow P \rightarrow 0
$$

of Lie algebras induces an exact sequence

$$
K \wedge M \rightarrow M \wedge M \rightarrow P \wedge P \rightarrow 0 .
$$

Again, this analogue is obtained using the identity $m \wedge n=-n \wedge m$ which holds in $M \wedge M$. We can say more if $M \rightarrow P$ is split.

Proposition 13. A split short exact sequence of Lie algebras

$$
0 \rightarrow K \rightarrow M \leftrightarrows P \rightarrow 0
$$

induces a natural split short exact sequence

$$
0 \rightarrow K \wedge M \rightarrow M \wedge M \leftrightarrows P \wedge P \rightarrow 0 .
$$

Proof. We just need to prove the injectivity of $K \wedge M \stackrel{\iota}{\rightarrow} \wedge \wedge M$. We do this by constructing a Lie homomorphism $\phi: M \wedge M \rightarrow(K \wedge M) \rtimes(P \wedge P)$ such that $\phi \iota$ is the canonical inclusion. Here $x \in P \wedge P$ acts on $(k \wedge m) \in K \wedge M$ by ${ }^{x}(k \wedge m)=([\partial x, k] \wedge m)$ $+(k \wedge[\partial x, m])$, where $\partial: P \wedge P \rightarrow P$ and $P$ is considered as a subalgebra of $M$. Since $M$ $\cong K \rtimes P$ we can define $\phi:(K \rtimes P) \wedge(K \rtimes P) \rightarrow(K \wedge(K \rtimes P)) \rtimes(P \wedge P)$ by $\phi\left(\left(k_{1}, p_{1}\right)\right.$ $\left.\wedge\left(k_{2}, p_{2}\right)\right)=\left(k_{1} \wedge\left(k_{2}, p_{2}\right)-k_{2} \wedge\left(0, p_{1}\right),\left(p_{1} \wedge p_{2}\right)\right)$. A page of routine calculations shows that $\phi$ preserves the defining relations of the exterior product and is thus the required Lie homomorphism.

In order to study the relation between the Lie exterior product and the Lie tensor product, we use a slightly generalized version of J. H. C. Whitehead's universal quadratic functor, the generalization being due to [31].

Definition. [31] The universal quadratic functor $\Gamma$ is defined for any $\Lambda$-module $A$ to be the $\Lambda$-module $\Gamma(A)$ generated by the symbols $\gamma(a)$ with $a \in A$, subject to the relations

$$
\begin{aligned}
\lambda^{2} \gamma(a) & =\gamma(\lambda a), \\
\gamma(a+b+c)+\gamma(a)+\gamma(b)+\gamma(c) & =\gamma(a+b)+\gamma(a+c)+\gamma(b+c), \\
\gamma(\lambda a+b)+\lambda \gamma(a)+\lambda \gamma(b) & =\lambda \gamma(a+b)+\gamma(\lambda a)+\gamma(b),
\end{aligned}
$$

for all $\lambda, \lambda^{\prime} \in \Lambda, a, b, c \in A$. 
Consider the Lie subalgebra $M \times{ }_{P} N=\left\{(m, n): \partial(m)=\partial^{\prime}(n)\right\}$ of the direct sum $M \oplus N$. Recall the homomorphisms $\mu: M \otimes N \rightarrow M, v: M \otimes N \rightarrow N$ from Proposition 2 and let $\langle M, N\rangle=\{(\mu x, v x): x \in M \otimes N\}$. It is readily checked that $\langle M, N\rangle$ is an ideal in $M \times_{P} N$, and that the quotient $\left(M \times_{P} N\right) /\langle M, N\rangle$ is abelian. For $(m, n) \in M \times{ }_{P} N$ we shall write $\overline{m, n})$ to denote the coset $(m, n)+\langle M, N\rangle$.

Proposition 14. There is a natural exact sequence of Lie algebras

$$
\Gamma\left(\left(M \times_{P} N\right) /\langle M, N\rangle\right) \stackrel{\psi}{\rightarrow} M \otimes N \stackrel{\pi}{\rightarrow} M \wedge N \rightarrow 0
$$

where $\psi(\gamma(\overline{m, n}))=m \otimes n$, and $\pi(m \otimes n)=m \wedge n$.

Proof. Certainly $\pi$ is surjective. Now any element $x \in M \otimes N$ is of the form $x=\sum_{i} m_{i} \otimes n_{i}$, so

$$
\mu x \otimes v x=-\sum_{i}\left({ }^{\left(n_{i}\right)}\left(m_{i}\right) \otimes{ }^{\left(m_{i}\right)}\left(n_{i}\right)\right)+\sum_{i<j}\left(\left(^{\left(n_{i}\right)}\left(m_{i}\right) \otimes^{\left(m_{j}\right)}\left(n_{j}\right)\right)-\left({ }^{\left(n_{j}\right)}\left(m_{j}\right) \otimes{ }^{\left(m_{i}\right)}\left(n_{i}\right)\right)\right) .
$$

Applying relation (iv) of the Lie tensor product, we see that $\mu x \otimes v x=0$. Thus if $(m, n)=0 \in\left(M \times_{P} N\right) /(M, N\rangle$ then $\psi(\gamma(\overline{m, n}))=m \otimes n=0$. Certainly $\psi$ preserves the defining relations of $\Gamma(-)$, and thus $\psi$ is a $\Lambda$-module homomorphism. Clearly $\operatorname{lm} \psi=$ $M \square N$. But $M \square N$ is in the centre of $M \otimes N$, and so $\psi$ is a Lie homomorphism.

One consequence of Proposition 14 is that for any Lie algebra $P$ there is an exact sequence

$$
\Gamma\left(P^{\mathrm{ab}}\right) \rightarrow P \otimes P \rightarrow P \wedge P \rightarrow 0 .
$$

More generally, if $M$ and $N$ are ideals of $P$, there is an exact sequence

$$
\Gamma((M \cap N) /[M, N]) \rightarrow M \otimes N \rightarrow M \wedge N \rightarrow 0 .
$$

From [31] we recall the following two results.

Proposition 15. [31] For any $\Lambda$-modules $A$ and $B$ there is an isomorphism

$$
\Gamma(A \oplus B) \cong \Gamma(A) \oplus \Gamma(B) \oplus(A \underset{\bmod }{\otimes} B) .
$$

Proposition 16. [31] Let I be a well-ordered set. If $A$ is a free $\Lambda$-module with basis $\left\{e_{i}\right\}_{i \in l}$, then $\Gamma(A)$ is a free $\Lambda$-module with basis

$$
\left\{\gamma\left(e_{i}\right)\right\}_{i \in l} \cup\left\{\omega\left(e_{i}, e_{j}\right)=\gamma\left(e_{i}+e_{j}\right)-\gamma\left(e_{i}\right)-\gamma\left(e_{j}\right)\right\}_{i<j} .
$$

In particular there is an isomorphism $\Gamma(\Lambda) \cong \Lambda$.

Proposition 17. If $P$ is a Lie algebra such that $P^{\mathrm{ab}}$ is a free $\Lambda$-module, then the sequence

$$
0 \rightarrow \Gamma\left(P^{\mathrm{ab}}\right) \stackrel{\psi}{\rightarrow} P \otimes P \stackrel{\pi}{\rightarrow} P \wedge P \rightarrow 0
$$

is exact.

Proof. We have only to prove that $\psi: \Gamma\left(P^{a b}\right) \rightarrow P \otimes P, \gamma(p+[P, P]) \mapsto p \otimes p$ is injective. Let $\theta: P \otimes P \rightarrow P^{\mathrm{ab}} \otimes P^{\mathrm{ab}}$ be the Lie homomorphism induced by the projection $P \rightarrow P^{\mathrm{ab}}$. Now $P^{\mathrm{ab}} \otimes P^{\mathrm{ab}} \cong P^{\mathrm{ab}} \bigotimes_{\mathrm{mod}} P^{\mathrm{ab}}$ is a free $\Lambda$-module, and so too is $\Gamma\left(P^{\mathrm{ab}}\right)$. Moreover 
the composite homomorphism $\theta \psi$ maps a basis of $\Gamma\left(P^{\mathrm{ab}}\right)$ injectively to part of a basis of $P^{\mathrm{ab}} \otimes P^{\mathrm{ab}}$. Thus $\theta \psi$ is injective. It follows that $\psi$ is injective.

Note that if $\Lambda$ is a field, then any Lie algebra $P$ over $\Lambda$ satisfies the hypothesis of this last proposition.

In [15] a natural isomorphism

$$
H_{2}(P) \cong \operatorname{ker}(P \wedge P \rightarrow P) .
$$

was obtained for any Lie algebra $P$. Consequently, for any free Lie algebra $F$ there is an isomorphism $F \wedge F \cong[F, F]$, since $H_{2}(F)$ is trivial.

Definition. For any Lie algebra $P$ we set

$$
J_{2}(P)=\operatorname{ker}\left(P \otimes P \rightarrow P, p \otimes p^{\prime} \mapsto\left[p, p^{\prime}\right]\right) .
$$

In Section 4 we obtain (a more general version of) the following theorem (see Theorem 27).

Theorem 18. For any Lie algebra $P$ there is a natural exact sequence

$$
H_{3}(P) \rightarrow \Gamma\left(P^{\mathrm{ab}}\right) \stackrel{\psi}{\rightarrow} J_{2}(P) \rightarrow H_{2}(P) \rightarrow 0,
$$

where the homomorphism $\psi$ is injective if $P^{\mathrm{ab}}$ is a free $\Lambda$-module.

We can use Theorem 18 to study $J_{2}(P * Q)$ where $P * Q$ is the free product of Lie algebras $P$ and $Q$.

Proposition 19. For any Lie algebras $P$ and $Q$ there is an isomorphism

$$
J_{2}(P * Q) \cong J_{2}(P) \oplus J_{2}(Q) \oplus\left(P^{\mathrm{ab}} \otimes Q^{\mathrm{ab}}\right) .
$$

Proof. Since $H_{n}(P * Q) \cong H_{n}(P) \oplus H_{n}(Q)$ and $\Gamma\left((P * Q)^{\mathrm{ab}}\right) \cong \Gamma\left(P^{\mathrm{ab}}\right) \oplus \Gamma\left(Q^{\mathrm{ab}}\right) \oplus$ $\left(P^{\mathrm{ab}} \otimes Q^{\mathrm{ab}}\right)$, we have a commutative diagram

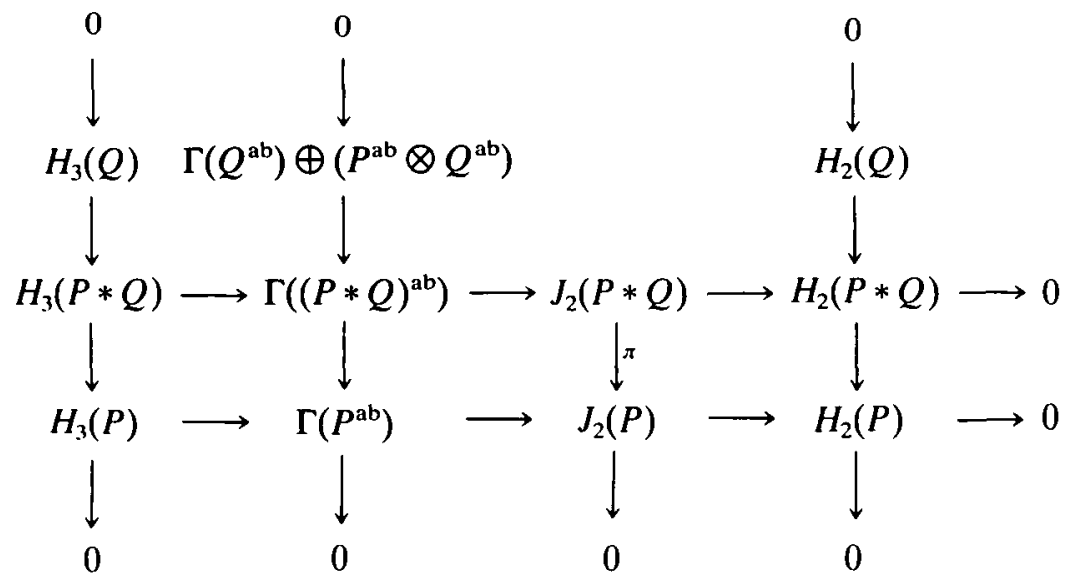

in which the columns are exact, the homomorphism $\pi$ being induced by the projection $P * Q \rightarrow P$. The rows are also exact by Theorem 18 . We thus have an exact sequence

$$
H_{3}(Q) \rightarrow \Gamma(Q) \oplus\left(P^{\mathrm{ab}} \otimes Q^{\mathrm{ab}}\right) \rightarrow \operatorname{ker}(\pi) \rightarrow H_{2}(Q) \rightarrow 0 .
$$


Now the projection $P * Q \rightarrow Q$ induces the split vertical maps in the commutative diagram

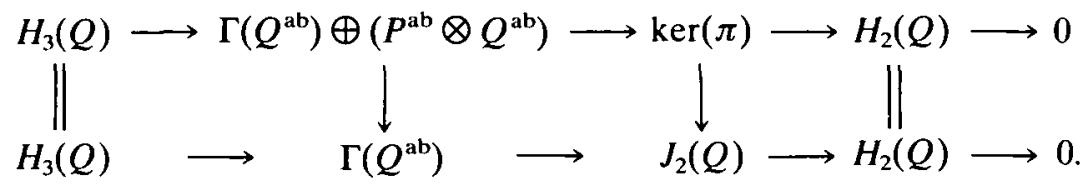

As the rows of this diagram are exact, we obtain an isomorphism $\operatorname{ker}(\pi) \cong J_{2}(Q) \oplus$ $\left(P^{\mathrm{ab}} \otimes P^{\mathrm{ab}}\right)$. But $\pi$ is split, and so $J_{2}(P * Q) \cong \operatorname{ker}(\pi) \oplus J_{2}(P)$. The isomorphism of the Proposition follows.

Corollary 20. For any Lie algebras $P_{1}, \ldots, P_{n}$ there is an isomorphism

$$
J_{2}\left(P_{1} * \ldots * P_{n}\right) \cong J_{2}\left(P_{1}\right) \oplus \ldots \oplus J_{2}\left(P_{n}\right) \oplus\left(\sum_{1 \leq i<j \leq n} P_{i}^{\mathrm{ab}} \otimes P_{j}^{\mathrm{ab}}\right) .
$$

The following formula for $J_{2}(P \oplus Q)$, where $P \oplus Q$ is the direct sum of Lie algebras $P$ and $Q$, is easily derived from Proposition 8.

Proposition 21. For any Lie algebras $P$ and $Q$ there is an isomorphism

$$
J_{2}(P \oplus Q) \cong J_{2}(P) \oplus J_{2}(Q) \oplus\left(P^{\mathrm{ab}} \otimes Q^{\mathrm{ab}}\right) \oplus\left(Q^{\mathrm{ab}} \otimes P^{\mathrm{ab}}\right) .
$$

3. Free and projective crossed modules. We now investigate a relationship between the Lie exterior product and free (and projective) crossed modules.

By the category of crossed $P$-modules we mean the category whose objects are crossed modules $\partial: M \rightarrow P$ with $P$ a fixed Lie algebra; in this category the morphisms from $\partial: M \rightarrow P$ to $\partial^{\prime}: M^{\prime} \rightarrow P$ are the $P$-equivariant Lie homomorphisms $\theta: M \rightarrow M^{\prime}$ such that $\partial^{\prime} \theta=\partial$. A projective object in this category will be called a projective crossed $P$-module.

It is readily seen that a projective $P$-module (in the usual sense) is one example of a projective crossed $P$-module. We can obtain more examples by considering "free" objects in the category of crossed $P$-modules. In fact there are three distinct notions of "freeness" to be considered.

In the following definition we say that $\delta: Q \rightarrow P$ is a $P$-homomorphism to mean that $Q$ is a $P$-module and $\delta$ is a $P$-module homomorphism with $P$ considered as a left $P$-module.

Definition. Let $S(A, Q)$ be a set $(\Lambda$-module, $P$-module) and let $\delta: S \rightarrow P(\delta: A \rightarrow$ $P, \delta: Q \rightarrow P$ ) be a set mapping ( $\Lambda$-module homomorphism, $P$-homomorphism). We say that a crossed $P$-module $\partial: M \rightarrow P$ is free on $\delta$ if:

(a) $S(A, Q)$ is a subset (sub $\Lambda$-module, sub $P$-module) of $M$;

(b) for any crossed module $\partial^{\prime}: M^{\prime} \rightarrow P$, any set mapping $v: S \rightarrow M^{\prime}$ ( $\Lambda$-module homomorphism $v: A \rightarrow M^{\prime}, P$-homomorphism $\left.v: Q \rightarrow M^{\prime}\right)$ satisfying $\partial^{\prime} v=\delta$ extends uniquely to a morphism $v: M \rightarrow M^{\prime}$ of crossed $P$-modules.

Clearly a free crossed $P$-module (in any of the three senses) is uniquely determined up to isomorphism by $\delta$.

It is readily seen that a crossed $P$-module is projective if it is free on some set mapping $\delta$. Also, the free crossed module on a $\Lambda$-module mapping $\delta: A \rightarrow P$ is a projective crossed $P$-module if $A$ is a projective $\Lambda$-module. Note that if $\Lambda$ is a field then every $\Lambda$-module is projective. 
For each of the three notions of freeness we now show how to construct a free crossed $P$-module.

Construction 1. Suppose that $\delta: Q \rightarrow P$ is a $P$-homomorphism. Let $\mathscr{A}^{1}(Q)=Q$ and $\mathscr{A}^{k}(Q)=\bigoplus_{0<i<k} \mathscr{A}^{i}(Q) \bigotimes_{\text {mod }} \mathscr{A}^{k-i}(Q)$. Then the module action of $P$ on $Q$ yields a $P$-module structure on $\mathscr{A}^{k}(Q)$ : if $x \otimes y \in \mathscr{A}^{i}(Q) \bigotimes_{\text {mod }} \mathscr{A}^{k-i}(Q)$ and $p \in P$ then, inductively, we define

$$
p(x \otimes y)=p x \otimes y+x \otimes p y,
$$

and this extends linearly to an action of $p$ on an arbitrary element of $\mathscr{A}^{k}(Q)$. Now let $\mathscr{A}(Q)=\bigoplus_{0<k} \mathscr{A}^{k}(Q)$. The action of $P$ on $\mathscr{A}^{k}(Q)$ extends linearly to an action of $P$ on $\mathscr{A}(Q)$, making $\mathscr{A}(Q)$ into a $P$-module. The inclusion maps $\mathscr{A}^{j}(Q) \bigotimes_{\bmod } \mathscr{A}^{k}(Q) \rightarrow \mathscr{A}^{j+k}(Q)$ give rise to a non-associative multiplication on $\mathscr{A}(Q)$.

Let $\mathscr{I}(Q)$ be the two-sided ideal of $\mathscr{A}(Q)$ generated by the elements

$$
x x \text { and } x(y z)+y(z x)+z(x y),
$$

where $x, y, z \in \mathscr{A}(Q)$. It is easily checked that $\mathscr{I}(Q)$ is $P$-invariant. We have a Lie algebra $\mathscr{L}(Q)=\mathscr{A}(Q) / \mathscr{I}(Q)$, which is well-known to be the free Lie algebra on the $\Lambda$-module $Q$. The action of $P$ on $\mathscr{A}(Q)$ induces a Lie action of $P$ on $\mathscr{L}(Q)$.

Now the $P$-homomorphism $\delta: Q \rightarrow P$ induces a unique Lie homomorphism ว: $\mathscr{L}(Q) \rightarrow P$ and, since $\delta$ is $P$-equivariant, $\partial$ is $P$-equivariant. Take $\mathscr{I}(Q)$ to be the ideal of $\mathscr{L}(Q)$ generated by the elements $[x, y]-{ }^{2 x} y$ for $x, y \in \mathscr{L}(Q)$. (By analogy with the group theoretic situation, we shall refer to $\mathscr{I}(Q)$ as the Peiffer ideal.) Since $\mathscr{F}(Q)$ is $P$-invariant, if we set $\mathscr{C}(Q)=\mathscr{L}(Q) / \mathscr{I}(Q)$, the induced homomorphism $\partial: \mathscr{C}(Q) \rightarrow P$ is a crossed module; clearly this is the free crossed module on $\delta: Q \rightarrow P$.

Construction 2. Suppose that $\delta: A \rightarrow P$ is a $\Lambda$-module homomorphism. Let $Q=$ $P^{e} \bigotimes_{\bmod } A$, where $P^{e}$ is the universal enveloping algebra of $P$. Thus $Q$ is a $P$-module, and there is a $P$-homomorphism $\delta^{\prime}: Q \rightarrow P, x \otimes a \mapsto x .(\delta a)$; here $x \in P^{c}, a \in A$ and we are using the fact that $P$ is a $P^{e}$-module. Construction 1 now provides us with the free crossed $P$-module $\mathscr{C}(Q) \rightarrow P$ on $\delta^{\prime}$, which is clearly also the free crossed $P$-module on $\delta$.

Construction 3. Suppose that $\delta: S \rightarrow P$ is a set map. Let $A$ be the free $\Lambda$-module on $S$, and let $\delta^{\prime \prime}: A \rightarrow P$ be the $\Lambda$-module homomorphism induced by $\delta$. Using Construction 2 we get the free crossed module $\mathscr{C}(Q) \rightarrow P$ on $\delta^{\prime \prime}$, where $Q=P^{e} \bigotimes_{\bmod } A$; this is clearly the free crossed module on $\delta$.

THEOREM 22. Suppose that $\Lambda$ is a field. Let $\partial: M \rightarrow P$ be a projective crossed $P$-module. Denote the image of $\partial$ by $\bar{M}$. Then the restricted homomorphism $\partial: M \rightarrow \bar{M}$ is a projective crossed $\bar{M}$-module.

Proof. Standard arguments show that a crossed $P$-module $\partial: M \rightarrow P$ is projective if and only if there is some free crossed $P$-module $\partial: \mathscr{C}(S) \rightarrow P$ on a set mapping $S \rightarrow P$ together with a pair of crossed $P$-module morphisms $\phi: \mathscr{C}(S) \rightarrow M, \sigma: M \rightarrow \mathscr{C}(S)$ such that $\phi \sigma$ is the identity. Thus we need only prove that for any such crossed $P$-module 
$\partial: \mathscr{C}(S) \rightarrow P$, the restricted crossed $\bar{M}$-module $\partial: \mathscr{C}(S) \rightarrow \bar{M}$ is free on some set mapping $\rho: R \rightarrow \bar{M}$.

So suppose that $\partial: \mathscr{C}(S) \rightarrow P$ is a free crossed module on a set mapping $\delta: S \rightarrow P$, and that $\bar{M}$ is the image of $\partial$. Since $\Lambda$ is a field we can consider $P^{e}$ as a free $\bar{M}$-module with basis $B$ say (see for example [22, Chapter VII, Corollary 1.4]). Let $R=B \times S$ and define $\rho: R \rightarrow \bar{M}$ by $\rho(b, s)=b .(\delta s)$ for $b \in B, s \in S$, where we are considering the ideal $\bar{M}$ of $P$ as a left $P^{e}$-module. If $A$ is the vector space over $\Lambda$ with basis $S$, then $P^{e} \bigotimes_{\text {mod }} A$ is the free $\bar{M}$-module on the set $R$. Hence $\rho$ induces an $\bar{M}$-module homomorphism $\rho^{\prime}: P^{e} \bigoplus_{\text {mod }} A \rightarrow \bar{M}$ which, in fact, is $P$-equivariant. Now $\rho^{\prime}$ induces an $\bar{M}$-equivariant Lie algebra homomorphism $\rho^{\prime \prime}: \mathscr{L}\left(P^{e} \bigotimes_{\bmod } A\right) \rightarrow \bar{M}$. By Construction $1, \quad \mathscr{C}(S)=\mathscr{L}\left(P^{e} \bigotimes_{\bmod } A\right) / \mathscr{I}(S)$ where $\mathscr{S}(S)$ is the ideal generated by the elements $[x, y]-\rho^{*} x y$ for $x, y \in \mathscr{L}\left(P^{e} \bigotimes_{\bmod } A\right)$.

Let $\partial^{\prime}: M^{\prime} \rightarrow \bar{M}$ be any crossed $\bar{M}$-module. Any set map $v: R \rightarrow M^{\prime}$ satisfying $\partial^{\prime} v=\rho$ induces a unique $\bar{M}$-module homomorphism $v^{\prime}: P^{c} \underset{\bmod }{\bigotimes} A \rightarrow M^{\prime}$ satisfying $\partial^{\prime} v^{\prime}=\rho^{\prime}$. The map $v^{\prime}$ induces an $\bar{M}$-equivariant homomorphism $\mathscr{L}\left(P^{e} \bigotimes_{\bmod } A\right) \rightarrow M^{\prime}$ which, since $M^{\prime} \rightarrow \bar{M}$ is a crossed module, in turn induces a homomorphism $v^{\prime \prime}: \mathscr{C}(S) \rightarrow M^{\prime}$; clearly $v^{\prime \prime}$ is a morphism of crossed $\bar{M}$-modules. This proves that $\partial: \mathscr{C}(S) \rightarrow \bar{M}$ is free on the set $\operatorname{map} \rho$.

Proposition 23. Let $\partial: M \rightarrow P$ be a projective crossed $P$-module with $\operatorname{Im}(\partial)=\bar{M}$ say. Then

(i) the ideal $[M, M]$ of $M$ is uniquely determined, up to isomorphism, by the Lie algebras $\bar{M}$ and $P$;

(ii) there is a surjective Lie homomorphism $\xi: \bar{M} \wedge \bar{M} \rightarrow[M, M]$.

Proof. (i) Suppose that $\partial^{\prime}: M^{\prime} \rightarrow P$ is another projective crossed $P$-module with $\operatorname{Im}\left(\partial^{\prime}\right)=\bar{M}$. Let $M \times_{P} M^{\prime}=\left\{\left(m, m^{\prime}\right) \in M \times M^{\prime}: \partial m=\partial^{\prime} m^{\prime}\right\}$. We have a homomorphism $M \times_{P} M^{\prime} \rightarrow P, \quad\left(m, m^{\prime}\right) \mapsto \partial m$, which we shall consider as a set mapping. Let $\mathscr{C}\left(M \times{ }_{P} M^{\prime}\right) \rightarrow P$ be the free crossed $P$-module on this set mapping. The projection $M \times{ }_{P} M^{\prime} \rightarrow M$ is surjective and induces a surjective morphism of crossed $P$-modules $v: \mathscr{C}\left(M \times{ }_{P} M^{\prime}\right) \rightarrow M$. It is readily checked that $v$ is a crossed $M$-module with $M$ acting on $\mathscr{C}\left(M \times{ }_{P} M^{\prime}\right)$ via $P$; hence $\operatorname{ker}(v)$ is in the centre of $\mathscr{C}\left(M \times{ }_{P} M^{\prime}\right)$. Since $M$ is projective there is a crossed $P$-module morphism $\sigma: M \rightarrow \mathscr{C}\left(M \times_{P} M^{\prime}\right)$ such that vo is the identity. Therefore $\mathscr{C}\left(M \times{ }_{P} M^{\prime}\right) \cong \operatorname{ker}(v) \oplus M$, and consequently $\left[\mathscr{C}\left(M \times{ }_{P} M^{\prime}\right)\right.$, $\left.\mathscr{C}\left(M \times_{P} M^{\prime}\right)\right] \cong[M, M]$. Similarly $\left[\mathscr{C}\left(M \times_{P} M^{\prime}\right), \mathscr{C}\left(M \times_{P} M^{\prime}\right)\right] \cong\left[M^{\prime}, M^{\prime}\right]$. Thus we have $[M, M] \cong\left[M^{\prime}, M^{\prime}\right]$.

(ii) Lemma 10 applied to the central extension $M \rightarrow \bar{M}$ yields a surjection $\bar{M} \otimes \bar{M} \rightarrow$ $[M, M]$, which in turn induces a surjection $\bar{M} \wedge \bar{M} \rightarrow[M, M]$.

We now give several characterizations of projective crossed modules.

THEOREM 24. Let $\partial: M \rightarrow P$ be a crossed $P$-module. Let $\bar{M}=\operatorname{Im}(\partial)$ and $\bar{P}=P / \bar{M}$. The following statements are equivalent if $\Lambda$ is a field or if $\bar{M}=P$.

(i) $\partial: M \rightarrow P$ is a projective crossed $P$-module.

(ii) $M^{\mathrm{ab}}$ with the induced action of $\bar{P}$ is a projective $\bar{P}$-module, and $\xi: \bar{M} \wedge \bar{M} \cong$ $[M, M]$ is an isomorphism. 
(iii) $M^{\text {ab }}$ is a projective $\bar{P}$-module and $H_{2}(\bar{M}) \cong \operatorname{ker}(\partial) \cap[M, M]$.

(iv) $M^{\mathrm{ab}}$ is a projective $\bar{P}$-module and the induced homomorphism $\partial_{*}: H_{2}(M) \rightarrow$ $\mathrm{H}_{2}(\bar{M})$ is trivial.

Proof. To prove (i) implies (ii), suppose that $\partial: M \rightarrow P$ is projective. Then we can find a free crossed $P$-module $\mathscr{C}(S) \rightarrow P$ on some set mapping $S \rightarrow P$ for which there is a split crossed $P$-module epimorphism $\phi: \mathscr{C}(S) \rightarrow M$. It is easily checked that $\phi: \mathscr{C}(S) \rightarrow M$ is a crossed $M$-module with $M$ acting on $\mathscr{C}(S)$ via $P$. Thus $\operatorname{ker}(\phi)$ is in the centre of $\mathscr{C}(S)$ and so there is a $P$-equivariant Lie isomorphism $\mathscr{C}(S) \cong \operatorname{ker}(\phi) \oplus M$. Note that $\operatorname{ker}(\phi)$ is a $\bar{P}$-module. It is readily seen that $\mathscr{C}(S)^{\mathrm{ab}} \cong \bar{P}^{e} \bigotimes_{\text {mod }} A$ where $A$ is the free $\Lambda$-module on $S$. Thus $\mathscr{C}(S)^{\mathrm{ab}}$ is a free $\bar{P}$-module. But $\mathscr{C}(S)^{\mathrm{ab}} \cong \operatorname{ker}(\phi) \oplus M^{\mathrm{ab}}$. It follows that $M^{\mathrm{ab}}$ is a projective $\bar{P}$-module.

The restricted crossed $\bar{M}$-module $\partial: M \rightarrow \bar{M}$ is projective if $\bar{M}=P$ (clearly) or if $\Lambda$ is a field (by Theorem 22). Now choose a presentation $R \hookrightarrow F \rightarrow \bar{M}$ of the Lie algebra $\bar{M}$, with $F$ the free Lie algebra on some set $S$. It is easily checked that the induced central extension $F /[F, R] \rightarrow \bar{M}$ is the free crossed $\bar{M}$-module on the set mapping $S \rightarrow \bar{M}$. Hence by Proposition 23(i) we have an isomorphism $[F /[F, R], F /[F, R]] \cong[M, M]$. But $[F /[F, R], F /[F, R]] \cong[F, F] /[F, R] \cong \bar{M} \wedge \bar{M}$. This proves that $\bar{M} \wedge \bar{M} \cong[M, M]$.

The isomorphism $H_{2}(\bar{M})=\operatorname{ker}(\bar{M} \wedge \bar{M} \rightarrow \bar{M})$ shows that (ii) implies (iii).

Now (iii) implies (iv) because the five term exact homology sequence (see [22] or Theorems 34,35 below) arising from the central extension $\operatorname{ker}(\partial) \leadsto M \stackrel{\partial}{\rightarrow} \bar{M}$ is

$$
H_{2}(M) \stackrel{\text {.. }}{\longrightarrow} H_{2}(\bar{M}) \rightarrow \operatorname{ker}(\partial) \stackrel{\iota}{\rightarrow} H_{1}(M) \rightarrow H_{1}(\bar{M}) \rightarrow 0,
$$

and $\operatorname{ker}(\iota)=\operatorname{ker}(\partial) \cap[M, M]$.

To prove that (iv) implies (i) suppose that $M^{\mathrm{ab}}$ is a projective $\bar{P}$-module and that $\partial_{*}$ is trivial. Let $\partial^{\prime}: E \rightarrow P$ be any crossed $P$-module. By standard arguments we see that $M$ is a projective crossed $P$-module if any surjective morphism of crossed $P$-modules $\varepsilon: E \rightarrow M$ splits. Certainly the induced morphism $\bar{\varepsilon}: E^{\mathrm{ab}} \rightarrow M^{\mathrm{ab}}$ yields an isomorphism $E^{\mathrm{ab}} \cong \operatorname{ker}(\bar{\varepsilon}) \oplus M^{\mathrm{ab}}$, and consequently a surjection $E \rightarrow E^{\mathrm{ab}} \rightarrow \operatorname{ker}(\bar{\varepsilon})$. The projection $E \rightarrow E^{\mathrm{ab}}$ induces a surjection $\pi: \operatorname{ker}(\varepsilon) \rightarrow \operatorname{ker}(\bar{\varepsilon})$. We shall show that $\pi$ is injective, and the resulting surjection $E \rightarrow E^{\mathrm{ab}} \rightarrow \operatorname{ker}(\bar{\varepsilon}) \cong \operatorname{ker}(\varepsilon)$ will give the desired splitting of $\varepsilon$. Note that the central extensions $\operatorname{ker}\left(\partial^{\prime}\right) \hookrightarrow E \rightarrow \bar{M}$ and $\operatorname{ker}(\partial) \nrightarrow M \rightarrow \bar{M}$ give rise to a commutative diagram

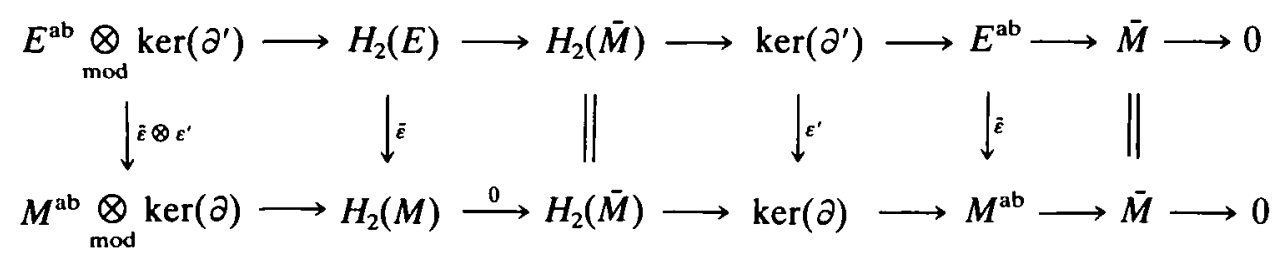

in which the rows are exact and are due to [15, Section 3, Remark (i)]. (See also Propositions 34 and 35 below.) In this diagram $\varepsilon^{\prime}$ and $\bar{\varepsilon}$ are surjective, and it follows that $\bar{\varepsilon}$ is surjective. The injectivity of $\pi$ follows from the exact sequence

$$
H_{2}(E) \stackrel{\tilde{\varepsilon}}{\rightarrow} H_{2}(M) \rightarrow \operatorname{ker}(\varepsilon) \rightarrow E^{\mathrm{ab}} \rightarrow M^{\mathrm{ab}} \rightarrow 0 .
$$


Recall that a Lie algebra $M$ is superperfect if $H_{1}(M)=H_{2}(M)=0$. From Theorem 24 we see that if $M$ is a superperfect ideal in some Lie algebra $P$, then the inclusion $M \hookrightarrow P$ is a projective crossed $P$-module. One example of such an ideal is $\operatorname{sl}(\Lambda)$, the ideal of matrices with zero trace in the Lie algebra $g l(\Lambda)$ of matrices over $\Lambda$ (see [26]).

4. Non-abelian derived functors. Let $\mathscr{F}: \mathscr{L}_{i e} \rightarrow \mathscr{L}_{i e}$ be the endofunctor on the category of Lie algebras which sends a Lie algebra $P$ to the free Lie algebra on the underlying set of $P$. We set $\mathscr{F}^{0}=\mathrm{id}: \mathscr{L}_{i e} \rightarrow \mathscr{L}_{i e}, \mathscr{F}^{1}=\mathscr{F}$ and $\mathscr{F}^{n}=\mathscr{F}_{\mathscr{F}^{n-1}}$ for $n \geq 2$. For $1 \leq i \leq n$ let $\varepsilon_{i}^{n}: \mathscr{F}^{n} P \rightarrow \mathscr{F}^{n-1} P$ denote the Lie homomorphism obtained by applying $\mathscr{F}^{i-1}$ to the canonical "augmentation map" $\mathscr{F}\left(\mathscr{F}^{n-i} P\right) \rightarrow \mathscr{F}^{n-i}(P)$. Then

$\mathscr{F} * P$ :

$$
\ldots \mathscr{F}^{n} P \underset{\varepsilon_{1}^{n}}{\stackrel{\varepsilon_{n}^{n}}{!}} \mathscr{F}^{n-1} P \ldots \underset{\varepsilon_{1}^{2}}{\stackrel{\varepsilon_{2}^{2}}{\longrightarrow}} \mathscr{F}^{1} P
$$

is a simplicial Lie algebra (in which we have not made explicit the degeneracy maps as they will not be needed).

Let $\mathscr{T}: \mathscr{L}_{i e} \rightarrow \mathscr{L}_{i e}$ be any endofunctor. Applying $\mathscr{T}$ dimension-wise to $\mathscr{F}^{*} P$ yields a simplicial Lie algebra:

$\mathscr{T} \mathscr{F}^{*} P$

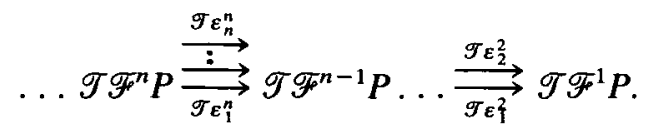

The $n$th homotopy group of $\mathscr{T}_{\mathscr{F}^{*}}(-)$ is called the $n$th derived functor of $\mathscr{T}$ and in this article it is denoted $\mathscr{D} \mathscr{T}_{n}(-)$. To be more explicit, recall that the homotopy groups of $\mathscr{T} \mathscr{F} * P$ are the homology groups of the Moore complex

$$
\mathcal{M} \mathscr{T}_{*}(P): \ldots \rightarrow \mathcal{M} \mathscr{T}_{n}(P) \stackrel{d_{n}}{\longrightarrow} \mathcal{M} \mathscr{T}_{n-1}(P) \rightarrow \ldots \stackrel{d_{1}}{\longrightarrow} \mathcal{M} \mathscr{T}_{0}(P) \stackrel{d_{0}}{\longrightarrow} 0
$$

where $\mathcal{M F}_{0}(P)=\mathscr{T} \mathscr{F}^{1} P, \mathcal{M} \mathscr{T}_{n}(P)=\bigcap_{1 \leq i \leq n} \operatorname{ker}\left(\mathscr{T}_{i}^{n+1}\right)$, and $d_{n}$ is the restriction of $\mathscr{T} \varepsilon_{n+1}^{n+1}$. Hence

$$
\mathscr{D} \mathscr{T}_{n}(P)=\operatorname{ker}\left(d_{n}\right) / \operatorname{Im}\left(d_{n+1}\right), \quad n \geq 0 .
$$

For more information on simplical Lie algebras see [8]. See [3] and [25] for more information on derived functors.

We denote the $n$th homology of a Lie algebra $P$ with coefficients in $\Lambda$ by $H_{n}(P)$. This homology can be described in terms of the derived functors of the endofunctor $(-)^{\mathrm{ab}}: \mathscr{L}_{i e} \rightarrow \mathscr{L}_{i e}, P \mapsto P^{\mathrm{ab}}$.

THEOREM 25 (Barr-Beck [3]). There is a natural isomorphism

for $n \geq 0$.

$$
H_{n+1}(P) \cong \mathscr{D}(-)_{n}^{\mathrm{ab}}(P)
$$

Proof. As pointed out in [29, Chapter II, Section 5], the cotriple description of group cohomology given in [2] carries over to the case of Lie algebra cohomology. Hence the proof of the cotriple description of group homology given in [3] carries over to the description of Lie algebra homology given in the theorem. 
Consider the endofunctor $\wedge^{2}: \mathscr{L}_{i e} \rightarrow \mathscr{L}_{i e}$ which sends a Lie algebra $M$ to the exterior product square $M \wedge M$.

Proposition 26. There are natural isomorphisms

$$
\begin{aligned}
& \mathscr{D} \wedge_{n}^{2}(P) \cong H_{n+2}(P) \quad \text { for } n \geq 1, \\
& \mathscr{D} \wedge_{0}^{2}(P) \cong P \wedge P .
\end{aligned}
$$

Proof. Since there is a simplicical isomorphism $\left[\mathscr{F}^{*} P, \mathscr{F}^{*} P\right] \cong \wedge^{2} \mathscr{F}^{*} P$, we have a short exact sequence

$$
0 \rightarrow \wedge^{2} \mathscr{F}^{*} P \rightarrow \mathscr{F}^{*} \rightarrow\left(\mathscr{F}^{*} P\right)^{\mathrm{ab}} \rightarrow 0
$$

of simplical Lie algebras. This gives rise to a long exact homotopy sequence

$$
\rightarrow 0 \rightarrow H_{n+2}(P) \rightarrow \mathscr{D} \wedge_{n}^{2}(P) \rightarrow 0 \rightarrow H_{n+1}(P) \rightarrow \ldots \rightarrow \mathscr{D} \wedge_{1}^{2}(P) \rightarrow P \rightarrow P^{\mathrm{ab}},
$$

from which the first isomorphism can be deduced.

Let $\mathscr{K} P$ be the kernel of $\varepsilon_{2}^{2}: \mathscr{F}^{2} P \rightarrow \mathscr{F}^{1} P$. By the exterior analogue of Proposition 9 we see that the kernel of the homomorphism $\varepsilon_{2}^{2} \wedge \varepsilon_{2}^{2}: \mathscr{F}^{2} P \wedge \mathscr{F}^{2} P \rightarrow \mathscr{F}^{1} P \wedge \mathscr{F}^{1} P$ is generated by the elements $x \wedge k$ with $x \in \mathscr{F}^{2} P$ and $k \in \mathscr{K} P$. It follows that the image of the homomorphism $d_{1}: \mathscr{K} P \rightarrow \mathscr{F}^{1} P \wedge \mathscr{F}^{1} P$ (where $d_{1}$ is the restriction of $\varepsilon_{1}^{2} \wedge \cdot \varepsilon_{1}^{2}$ ) is generated by the elements $\bar{x} \wedge \bar{k}$ where $\bar{x} \in \mathscr{F}^{1} P$ and $\bar{k} \in \varepsilon_{2}^{2}(\mathscr{K} P)$. Now $\mathscr{F}^{1} P / \varepsilon_{2}^{2}(\mathscr{K} P) \cong P$. It follows from the exterior analogue of Proposition 9 that $\mathscr{D} \wedge_{0}^{2}(P)=\operatorname{coker}\left(d_{1}: \mathscr{K} P \rightarrow \mathscr{F}^{1} P \wedge\right.$ $\left.\mathscr{F}^{1} P\right) \cong P \wedge P$.

We shall now prove a generalized version of Theorem 18. To do this we define Lie algebras $J_{n}(P)$ and $\Gamma_{n}(P)$ for $n \geq 2$.

Let $\otimes^{2}: \mathscr{L}_{i e} \rightarrow \mathscr{L}_{i e}$ be the endofunctor which maps $M$ to the tensor square $M \otimes M$. We write $J_{n+2}(P)=\mathscr{D} \otimes_{n}^{2}(P)$ for $n \geq 0$. Note that, analogous to the second isomorphism in Proposition 26, there is an isomorphism $\mathscr{D} \otimes_{0}^{2}(P) \cong P \otimes P$.

Let $\Gamma^{\text {ab }}: \mathscr{L}_{i e} \rightarrow \mathscr{L}_{i e}$ the endofunctor which maps $M$ to $\Gamma\left(M^{\mathrm{ab}}\right)$. We write $\Gamma_{n+2}(P)=$ $\mathscr{D} \Gamma_{n}^{\mathrm{ab}}(P)$ for $n \geq 0$. Analogous to the second isomorphism in Proposition 26 , there is an isomorphism $\mathscr{D} \Gamma_{0}^{\mathrm{ab}}(P) \cong \Gamma\left(P^{\mathrm{ab}}\right)$.

THEOREM 27. For any Lie algebra $P$ there is a natural long exact sequence

$$
\rightarrow \Gamma_{3}(P) \rightarrow J_{3}(P) \rightarrow H_{3}(P) \rightarrow \Gamma\left(P^{\mathrm{ab}}\right) \stackrel{\Psi}{\rightarrow} J_{2}(P) \rightarrow H_{2}(P) \rightarrow 0,
$$

where $\psi$ is injective if $P^{\mathrm{ab}}$ is a free $\Lambda$-module.

Proof. By Proposition 17 we have a short exact sequence of simplicial Lie algebras

$$
0 \rightarrow \Gamma\left(\mathscr{F}^{*} P\right)^{\mathrm{ab}} \rightarrow \mathscr{F}^{*} P \otimes \mathscr{F}^{*} P \rightarrow \mathscr{F}^{*} P \wedge \mathscr{F}^{*} P \rightarrow 0,
$$

the long exact homotopy sequence of which is, by Theorem 25 and Proposition 26, the required sequence.

We know (by virtue of identity (iv) of the Lie tensor product) that if $P$ is a perfect Lie algebra then $P \otimes P \cong P \wedge P$ and hence that $J_{2}(P) \cong H_{2}(P)$. We can extend this isomorphism to higher dimensions so as to get a sort of "Hurewicz theorem". 
THEOREM 28. Let $n \geq 2$ and let $P$ be a Lie algebra such that

(i) $H_{i}(P)=0$ for $2 \leq i \leq n-1$,

(ii) $H_{1}(P)=0$ if $n=2$ or 3 ,

(iii) $H_{1}(P)$ is free abelian (possibly $H_{1}(P)=0$ ) if $n \geq 4$.

Then the canonical maps $J_{n}(P) \stackrel{\cong}{\rightarrow} H_{n}(P), J_{n+1}(P) \rightarrow H_{n+1}(P)$ are respectively an isomorphism and a surjection.

Proof. We see that the free simplical $\Lambda$-module $\left(\mathscr{F}^{*} P\right)^{\text {ab }}$ obtained from $\mathscr{F}^{*} P$ by abelianizing dimension-wise has $i$ th homotopy group equal to $H_{i+1}(P)$. Thus by modifying $\left(F^{*} P\right)^{\text {ab }}$ only in dimensions greater than or equal to $n$ we can convert it into a free simplicial $\Lambda$-module resolution of $H_{1}(P)$. Now the simplicial module obtained from $\left(\mathscr{F}^{*} P\right)^{\text {ab }}$ by applying $\Gamma(-)$ dimension-wise has $i$ th homotopy group equal to $\Gamma_{i+2}(P)$. Hence $\Gamma_{n}(P)=\Gamma_{n-1}(P)=0$. Thus Theorem 27 implies the required isomorphism and surjection.

One immediate consequence of Theorem 28 is that the universal central extension $P \otimes P$ of a perfect Lie algebra $P$ is such that $\Gamma_{3}(P \otimes P)=0$ and $J_{3}(P \otimes P) \cong H_{3}(P \otimes P)$.

5. Relative derived functors. Suppose given a short exact sequence

$$
0 \rightarrow M \rightarrow P \stackrel{\phi}{\rightarrow} Q \rightarrow 0
$$

of Lie algebras. Then for any endofunctor $\mathscr{T}: \mathscr{L}_{i e} \rightarrow \mathscr{L}_{i e}$ the homomorphism $\phi$ induces a surjective simplical Lie homomorphism $\phi_{*}: \mathscr{T} \mathscr{F}^{*} P \rightarrow \mathscr{T F F}^{*} Q$. We define relative derived functors

$$
\mathscr{D} \mathscr{T}_{n}(P ; M)=\pi_{n}\left(\operatorname{ker} \phi_{*}\right)
$$

Since a short exact sequence of simplicial Lie algebras gives rise to a long exact homotopy sequence, we have immediately

LEMMA 29. There is a natural long exact sequence

$$
\rightarrow \mathscr{D} \mathscr{T}_{n+1}(Q) \rightarrow \mathscr{D} \mathscr{T}_{n}(P ; M) \rightarrow \mathscr{D T}_{n}(P) \rightarrow \mathscr{D} \mathscr{T}_{n}(Q) \rightarrow \ldots \rightarrow \mathscr{D} \mathscr{T}_{0}(Q) \rightarrow 0
$$

Now suppose given another short exact sequence of Lie algebras

$$
0 \rightarrow N \rightarrow P \stackrel{\Psi}{\rightarrow} R \rightarrow 0 \text {. }
$$

Then $\phi$ induces a surjection $\phi^{\prime}: R \rightarrow P /(M+N)$, which in turn induces a simplicial surjection $\phi_{*}^{\prime}: \mathscr{T} \mathscr{F}^{*} R \rightarrow \mathscr{T}^{*}(P /(M+N))$. The homomorphism $\psi$ induces a simplicial surjection $\psi_{*}^{\prime \prime}: \operatorname{ker} \phi_{*} \rightarrow \operatorname{ker} \phi_{*}^{\prime}$. We define the double relative derived functors as

$$
\mathscr{D} \mathscr{T}_{n}(P ; M, N)=\pi_{n} \operatorname{ker} \psi_{*}^{\prime \prime}
$$

Note that $\mathscr{D} \mathscr{T}_{n}(P ; M, N) \cong \mathscr{D} \mathscr{T}_{n}(P ; N, M)$. The long exact homotopy sequence gives us

LEMMA 30. There is a natural long exact sequence

$$
\begin{aligned}
\rightarrow \mathscr{D T}_{n+1}(P / N ; M+N / N) \rightarrow & \mathscr{D T}_{n}(P ; M, N) \rightarrow \mathscr{D T}_{n}(P ; M) \\
& \rightarrow \mathscr{D} \mathscr{T}(P / N ; M+N / N) \rightarrow \ldots \rightarrow \mathscr{D}_{0}(P / N ; M+N / N) \rightarrow 0 .
\end{aligned}
$$

The exact sequences of Lemmas 29 and 30 can be spliced together (compare [16, Proposition 3]) to give 
LEMMA 31. If $\mathscr{D T}_{n}(P /(M+N))=0$ for all $n \geq 0$ then there is a natural long exact sequence

$$
\begin{aligned}
\rightarrow \mathscr{D} \mathscr{T}_{n+1}(P / M) \oplus \mathscr{D} \mathscr{T}_{n+1}(P / N) \rightarrow \mathscr{D} \mathscr{T}_{n}(P ; M, N) & \rightarrow \mathscr{D} \mathscr{T}_{n}(P) \rightarrow \\
& \rightarrow \mathscr{D} \mathscr{T}_{n}(P / M) \oplus \mathscr{D T}_{n}(P / N) \rightarrow \ldots \rightarrow \mathscr{D} \mathscr{T}_{0}(P / M) \oplus \mathscr{D} \mathscr{T}_{0}(P / N) \rightarrow 0 .
\end{aligned}
$$

We shall now give computational descriptions of $\mathscr{D}_{0}(P ; M)$ and $\mathscr{D}_{0}(P ; M, N)$.

Consider the diagram

$$
(M \oplus M) \rtimes P \underset{q_{1}, q_{2}, q_{3}}{\stackrel{\longrightarrow}{\rightrightarrows}} M \rtimes P \stackrel{p_{2}}{\underset{p_{1}}{\longrightarrow}} P,
$$

where $\rtimes$ denotes a semi-direct product; the action of $P$ on $M$ is ${ }^{p} m=[p, m]$; the action of $P$ on $M \otimes M$ is ${ }^{p}\left(m, m^{\prime}\right)=\left([p, m],\left[p, m^{\prime}\right]\right)$; the homomorphisms are $p_{1}(m, p)=$ $m+p, \quad p_{2}(m, p)=p, \quad q_{1}\left(m^{\prime}, m, p\right)=\left(m^{\prime}-m, m+p\right), \quad q_{2}\left(m^{\prime}, m, p\right)=\left(m^{\prime}, p\right) \quad$ and $q_{3}\left(m^{\prime}, m, p\right)=(m, p)$.

Applying $\mathscr{D} \mathscr{T}_{0}$ to the above diagram we get the diagram

$$
\mathscr{D} \mathscr{T}_{0}((M \oplus M) \rtimes P) \underset{\overline{\bar{q}_{1}, \bar{q}_{2}, \vec{q}_{3}}}{\rightleftharpoons} \mathscr{D} \mathscr{T}_{0}(M \rtimes P) \underset{\bar{p}_{1}}{\stackrel{\bar{p}_{2}}{\longrightarrow}} \mathscr{D} \mathscr{T}_{0}(P),
$$

where we have written $\bar{q}_{i}$ and $\bar{p}_{i}$ instead of $\mathscr{D} \mathscr{T}_{0}\left(q_{i}\right)$ and $\mathscr{D} \mathscr{T}_{0}\left(p_{i}\right)$. With this notation we have

LeMma 32. There is an isomorphism

$$
\mathscr{D} \mathscr{T}_{0}(P ; M) \cong\left\{\operatorname{ker} \bar{p}_{2}\right\} /\left\{\bar{q}_{1}\left(\operatorname{ker} \bar{q}_{2} \cap \operatorname{ker} \bar{q}_{3}\right)\right\}
$$

Proof. This isomorphism is (a special case of the) Lie algebra version of [16, Lemma 9] (also compare [25, Theorem 9]). It is clear that we can replace "group" by "Lie algebra" throughout the proof of [16, Lemma 9].

Now the diagram

$$
(N \oplus N) \rtimes P \underset{q_{1}^{\prime}, q_{2}^{\prime} q_{3}^{\prime}}{\rightleftharpoons} N \rtimes P \underset{p_{1}^{\prime}}{\stackrel{p_{2}^{\prime}}{\longrightarrow}} P
$$

induces a diagram

$$
\begin{aligned}
\mathscr{D} \mathscr{T}_{0}((N \oplus N) \rtimes P ;(M \cap N) \oplus(M \cap N) \rtimes M) \underset{\overline{\bar{q}_{1}^{\prime}, \bar{q}_{2}^{\prime}, \bar{q}_{3}^{\prime}}}{\rightleftarrows} \mathscr{D} \mathscr{T}_{0}(N \rtimes P ;(M \cap N) \rtimes M) \\
\stackrel{\underset{\bar{p}_{1}^{\prime}}{\longrightarrow}}{\longrightarrow} \mathscr{T}_{0}(P ; M),
\end{aligned}
$$

and we have

LEMMA 33. There is an isomorphism

$$
\mathscr{D} \mathscr{T}_{0}(P ; M, N) \cong\left\{\operatorname{ker} \bar{p}_{2}^{\prime}\right\} /\left\{\bar{q}_{1}^{\prime}\left(\operatorname{ker} \bar{q}_{2}^{\prime} \cap \operatorname{ker} \bar{q}_{3}^{\prime}\right)\right\} \text {. }
$$

Proof. Let $\mathscr{S} \mathscr{L}_{i e}$ be the category whose objects are surjective Lie homomorphisms and whose morphisms are commutative squares of Lie homomorphisms. Let $\mathscr{T}^{r}: \mathscr{S}^{\mathscr{L}_{i e} \rightarrow}$ 
$\mathscr{L}_{i e}$ be the functor defined by $\mathscr{T}^{r}(P \rightarrow Q)=\operatorname{ker}(\mathscr{T} P \rightarrow \mathscr{T} Q)$. Then $\mathscr{D} \mathscr{T}_{n}(P ; M)$ can be considered as the $n$th derived functor of $\mathscr{T}^{r}$ (compare [16, Section 2]). Hence the isomorphism of the proposition follows from the Lie algebra version of [16, Lemma 9].

Definition. For ideals $M, N$ of $P$ we define the relative and double relative homology groups with coefficients in $\Lambda$ to be

for $n \geq 1$.

$$
\begin{gathered}
H_{n}(P ; M)=\mathscr{D}()_{n-1}^{\mathrm{ab}}(P ; M), \\
H_{n}(P ; M, N)=\mathscr{D}()_{n-1}^{\mathrm{ab}}(P ; M, N)
\end{gathered}
$$

Proposition 26 with Lemmas 29, 30, and 31 gives us

Theorem 34. For any ideals $M, N$ of a Lie algebra $P$ there are natural long exact sequences

and

$$
\rightarrow H_{n+1}(P / M) \rightarrow H_{n}(P ; M) \rightarrow H_{n}(P) \rightarrow H_{n}(P / M) \rightarrow \ldots \rightarrow H_{1}(P / M) \rightarrow 0
$$

$\rightarrow H_{n+1}(P / N ; M+N / N) \rightarrow H_{n}(P ; M, N) \rightarrow H_{n}(P ; M) \rightarrow H_{n}(P / N ; M+N / N)$

$$
\rightarrow \ldots \rightarrow H_{1}(P / N ; M+N / N) \rightarrow 0 .
$$

If moreover $H_{i}(P / M+N)=0$ for $i \geq 1$, then there is also a natural exact sequence

$\rightarrow H_{n+1}(P / M) \oplus H_{n+1}(P / N) \rightarrow H_{n}(P ; M, N) \rightarrow H_{n}(P) \rightarrow$

$$
\rightarrow H_{n}(P / M) \oplus H_{n}(P / N) \rightarrow \ldots \rightarrow H_{0}(P / M) \oplus H_{0}(P / N) \rightarrow 0 .
$$

The following descriptions of $H_{1}(P ; M), H_{1}(P ; M, N)$ and $H_{2}(P ; M)$ turn these exact sequences into computational tools.

THEOREM 35. For any ideals $M, N$ of a Lie algebra $P$ there are isomorphisms

(i) $H_{1}(P ; M) \cong P /[P, M]$,

(ii) $H_{1}(P ; M, N) \cong\{M \cap M\} /\{[P, M \cap N]+[M, N]\}$,

(iii) $H_{2}(P ; M) \cong \operatorname{ker}(P \wedge M \rightarrow P)$.

Proof. Proposition 26 and Lemma 32 give us an isomorphism

$$
H_{1}(P ; M) \cong \operatorname{ker}\left\{(M \rtimes P)^{\mathrm{ab}} \stackrel{\bar{p}_{2}}{\longrightarrow} P^{\mathrm{ab}}\right\},
$$

since for $\bar{q}_{i}:((M \oplus M) \rtimes P)^{\mathrm{ab}} \rightarrow(M \rtimes P)^{\mathrm{ab}}$ we clearly have ker $\bar{q}_{2} \cap \operatorname{ker} \bar{q}_{3}=0$. Isomorphism (i) follows.

Isomorphism (i) and Lemma 33 give us an isomorphism

$$
H_{1}(P ; M, N) \cong \operatorname{ker}\left\{((M \cap N) \rtimes M) /[N \rtimes P,(M \cap N) \rtimes M] \stackrel{\bar{p}_{2}}{\longrightarrow} M /[P, M]\right\}
$$

since for the appropriate $\bar{q}_{i}^{\prime}$ we have $\operatorname{ker} \bar{q}_{2}^{\prime} \cap \operatorname{ker} \bar{q}_{3}^{\prime}=0$. From this we get isomorphism (ii).

Consider the functor $\wedge^{2}: \mathscr{L}_{i e} \rightarrow \mathscr{L}_{i e}$. We have an isomorphism

$$
H_{2}(P ; M) \cong \operatorname{ker}\left(\mathscr{D} \wedge_{0}^{2}(P ; M) \rightarrow P\right) \text {. }
$$


So to obtain isomorphism (iii) we need to show that there is an isomorphism $\mathscr{D} \wedge{ }_{0}^{2}(P ; M) \cong P \wedge M$. Let $\bar{q}_{i}:(M \rtimes M \rtimes P) \wedge(M \rtimes M \rtimes P) \rightarrow(M \rtimes P) \wedge(M \rtimes P)$ and $\bar{p}_{i}:(M \rtimes P) \wedge(M \rtimes P) \rightarrow P \wedge P$ be the homomorphisms of Lemma 32. By the exterior analogue of Proposition 9 there is an exact sequence

$$
M \wedge(M \oplus M \rtimes P) \rightarrow(M \oplus M \rtimes P) \wedge(M \oplus M \rtimes P) \stackrel{\bar{q}_{i}}{\longrightarrow}(M \rtimes P) \wedge(M \rtimes P) \rightarrow 0
$$

for $i=2,3$; hence $\operatorname{ker} \bar{q}_{2}$ is generated by the elements $(m, 0,0) \wedge\left(m^{\prime}, m^{\prime \prime}, p\right)$, and ker $\bar{q}_{3}$ is generated by the elements $(0, m, 0) \wedge\left(m^{\prime}, m^{\prime \prime}, p\right)$. Thus ker $\bar{q}_{2} \cap \operatorname{ker} \bar{q}_{3}$ is generated by elements of the form $(m, 0,0) \wedge\left(0, m^{\prime}, 0\right)$. It follows that $\bar{q}_{1}\left(\operatorname{ker} \bar{q}_{2} \cap \operatorname{ker} \bar{q}_{3}\right)$ is generated by elements of the form $(m, 0) \wedge\left(-m^{\prime}, m^{\prime}\right)$. By Proposition 13 we see that $\operatorname{ker} \bar{p}_{2}=$ $(M \rtimes 0) \wedge(M \rtimes P)$. From the exterior analogue of Proposition 9 and Lemma 32 we get

$$
\mathscr{D} \wedge_{0}^{2}(P ; M) \cong\left\{\operatorname{ker} \bar{p}_{2}\right\} /\left\{\bar{q}_{1}\left(\operatorname{ker} \bar{q}_{2} \cap \operatorname{ker} \bar{q}_{3}\right)\right\} \cong M \wedge P \cong P \wedge M .
$$

A routine proof (analogous to the proof of [4, Theorem 1]) using the first two sequences of Theorem 34 and isomorphisms (i) and (ii) of Theorem 35 gives the following result.

THEOREM 36. Let $F$ be free Lie algebra with ideals $R, S$ such that $H_{i}(F / R)=$ $H_{i}(F / S)=0$ for $i=2,3$. Then there is an isomorphism

$$
H_{3}\left(\frac{F}{R+S}\right)=\frac{R \cap S \cap[F, F]}{[F, R \cap S]+[R, S]} .
$$

This isomorphism is a generalization of the well-known Hopf formula for the second homology of a Lie algebra. (See for example [22, Chapter VII, Exercise 3.2]).

\section{REFERENCES}

1. R. Aboughazi, Produit tensoriel du groupe d'Heisenberg, Bull. Soc. Math. France 115 (1987), 1-12.

2. M. Barr and J. Beck, Acyclic models and triples, in S. Eilenberg, D. K. Harrison, S. MacLane, H. Röhrl, ed., Proceedings of the conference on categorical algebra La Jolla 1965 (Springer, 1966), 344-354.

3. M. Barr and J. Beck, Homology and standard constructions, in B. Eckmann, ed., Seminar on triples and categorical homology theory, Lecture Notes in Mathematics 80 (Springer, 1969), 245-335.

4. R. Brown and G. J. Ellis, Hopf formulae for the higher homology of a group, Bull. London Math. Soc. 20 (1988), 124-128.

5. R. Brown, D. L. Johnson and E. F. Robertson, Some computations of nonabelian tensor products of groups, J. Algebra 111 (1987), 177-202.

6. R. Brown and J.-L. Loday, Van Kampen theorems for diagrams of spaces, Topology 26 (1987), 311-335.

7. R. Brown and J.-L. Loday, Homotopical excision, and Hurewicz theorems, for $n$-cubes of spaces, Proc. London Math. Soc. (3) 54 (1987), 176-192.

8. E. B. Curtis, Simplicial homotopy theorey, Adv. Math. 6 (1971), 107-204.

9. K. Dennis, In search of new homology functors having a close relationship to $K$-theory, preprint, 1976.

10. G. J. Ellis, Crossed modules and their higher dimensional analogues ( $\mathrm{Ph} . \mathrm{D}$. thesis, University of Wales, 1984). 
11. G. J. Ellis, Nonabelian exterior products of groups and an exact sequence in the homology of groups, Glasgow Math. J. 29 (1987), 13-19.

12. G. J. Ellis, The nonabelian tensor product of finite groups is finite, J. Algebra 111 (1987), 203-205.

13. G. J. Ellis, An algebraic derivation of a certain exact sequence, J. Algebra 117 (1989), $178-181$.

14. G. J. Ellis, On the higher universal quadratic functors and related computations (Galway Preprint, 1989).

15. G. J. Ellis, Nonabelian exterior products of Lie algebras and an exact sequence in the homology of Lie algebras, J. Pure Appl. Algebra 46 (1987), 111-115.

16. G. J. Ellis, Relative derived functors and the homology of groups, Cahiers Topologie Géom. Différentielle Catégoriques, to appear.

17. G. J. Ellis and T. Porter, Free and projective crossed modules and the second homology of a group, J. Pure Appl. Algebra 40 (1986), 27-31.

18. N. D. Gilbert, The nonabelian tensor square of a free group, Arch. Math. (Basel), 48 (1987), 369-375.

19. N. D. Gilbert and P. J. Higgins, The nonabelian tensor product of groups and related constructions, Glasgow Math. J. 31 (1989), 17-29.

20. D. Guin, "Cohomologie et homologie non abéliennes des groupes," J. Pure Appl. Algebra 50 (1988), 109-137.

21. D. Guin, Cohomologie non abélienne des algèbres de Lie (Strasbourg Preprint, 1987).

22. P. J. Hilton and U. Stammbach, $A$ course in homological algebra. Graduate Texts in Mathematics 4 (Springer, 1971).

23. D. L. Johnson, The nonabelian tensor square of a finite split metacyclic group, Proc. Edinburgh Math. Soc. (2) 30 (1987), 91-96.

24. F. J. Korkes, The cohomological and combinatorial group theory of profinite groups (Ph.D. thesis, University of Wales, 1986).

25. F. Keune, The relativization of $K_{2}$, J. Algebra 54 (1978), 159-177.

26. C. Kassel and J.-L. Loday, Extensions centrales d'algèbres de Lie, Ann. Inst. Fourier (Grenoble) 33 (1982), 119-142.

27. A. S.-T. Lue, The Ganea map for nilpotent groups, J. London Math. Soc. (2) 14 (1976), 309-312.

28. C. Miller, The second homology group of a group, Proc. Amer. Math. Soc. 3 (1952), $588-595$.

29. D. G. Quillen, Homotopical Algebra, Lecture Notes in Mathematics 43 (Springer, 1967). 66-74.

30. J. G. Ratcliffe, Free and projective crossed modules, J. London Math. Soc. (2) 22 (1980),

31. D. Simson and A. Tyc, Connected sequences of stable derived functors and their applications, Dissertationes Mathematicae (Rozprawy Mat.) 111 (1974).

Mathematics Department

University COLlege

GALWAY

IRELAND 